\title{
Prevalência de talassemias e hemoglobinas variantes em pacientes com anemia não ferropênica
}

\section{Prevalence of thalassemias and variant hemoglobins in patients with non-ferropenic anemia}

\author{
Sandrine C. Wagner ${ }^{1}$ \\ Matheus C. Silvestri ${ }^{2}$ \\ Christina M. Bittar ${ }^{2}$ \\ João R. Friedrisch ${ }^{2}$ \\ Lúcia M. R. Silla ${ }^{2}$
}

\begin{abstract}
Para estabelecer a freqüência de hemoglobinopatias e talassemias em pacientes com anemia não ferropênica foram estudados 58 casos de pacientes comprovadamente com anemia não ferropênica e 235 controles obtidos de pessoas sem anemia. Todas as amostras foram obtidas do Hospital de Clínicas de Porto Alegre (HCPA), RS, Brasil. As técnicas realizadas foram eletroforese em acetato de celulose, $p H$ alcalino, pesquisa citológica de $\mathrm{Hb} H, H P L C$, hemograma e ferritina. A análise dos dados realizada no grupo de pacientes com anemia não ferropênica demonstrou que $63,8 \%$ eram portadores de alguma forma de anemia hereditária: $25,9 \%$ de talassemia alfa heterozigota, $32,8 \%$ de talassemia beta heterozigota, 3,4\% de heterozigose para hemoglobina $\mathrm{S}$ ( $\mathrm{Hb}$ $A S)$ e 1,7\% de homozigose para hemoglobina $C$ ( $H b C C$ ). No grupo dos controles, foram identificados $14,1 \%$ de anemias hereditárias, sendo destas $11,5 \%$ de talassemia alfa, 0,9\% de talassemia beta, 1,3\% de heterozigose para hemoglobina $S$ (Hb AS) e $0,4 \%$ de heterozigose para hemoglobina $\mathrm{C}$ ( $\mathrm{Hb} \mathrm{AC}$ ). Os resultados obtidos permitem concluir que a prevalência de talassemias e hemoglobinas variantes no grupo controle é coincidente com a descrita na literatura. Entretanto, a excepcional prevalência dessas hemopatias hereditárias em pessoas com anemia não ferropênica deve ser divulgada entre médicos e serviços de saúde dada a sua importância no diagnóstico definitivo de anemia e dos corretos procedimentos terapêticos. Rev. bras. hematol. hemoter. 2005; 27(1):37-42.
\end{abstract}

Palavras-chave: Hemoglobinopatias; talassemia; anemia não ferropênica; epidemiologia.

\section{Introdução}

As anemias hereditárias estão entre as doenças genéticas mais comuns e compreendem um grupo de condições de variável complexidade. ${ }^{1}$ No passado, sua distribuição geográfica abrangia apenas áreas tropicais e subtropicais do mundo, cuja explicação se baseia no efeito protetor que tinham os portadores heterozigotos falcêmicos e talassêmicos contra as infecções endêmicas causadas pelos plasmódios da malária. Devido ao aumento dos movimentos migratórios ocorridos em diversas regiões, com conseqüente miscigena- ção, essas variantes acabaram-se por difundirem em áreas antes tidas como não endêmicas, como no Continente Americano e no Norte da Europa. ${ }^{2}$

Atualmente, mais de 1.100 mutações envolvendo os genes das cadeias globinas já foram descritas. ${ }^{3}$ Em maneira geral, as anemias hereditárias por defeito da hemoglobina podem ser divididas em dois grandes grupos: (1) as hemoglobinopatias, que se caracterizam pela presença de hemoglobinas estruturalmente anormais, tal como a hemoglobina $\mathrm{S}(\mathrm{Hb} \mathrm{S})$, que no estado homozigoto é responsável pela anemia falciforme, e (2) as talassemias, caracterizadas pela sínte-

\footnotetext{
IPrograma em Pós-Graduação em Medicina: Clínica Médica - UFRGS, Porto Alegre, Brasil.

${ }^{2}$ Centro de Atenção Global aos Portadores de Hemoglobinopatias do Serviço de Hematologia e Transplantes do Hospital de Clínicas de Porto Alegre (HCPA), Brasil.
}

Correspondência para: Sandrine C. Wagner

Rua Cel. Fernando Machado, 501/301 - Centro

90010-321 - Porto Alegre-RS - Brasil

Tel. 513227.1041

E-mail: swagner@adufrgs.ufrgs.br /wagnersandrine@hotmail.com 
se deficiente de uma ou mais cadeias polipeptídicas (globinas) das hemoglobinas humanas normais. Especialmente com referência às talassemias, elas podem ser classificadas em alfa $(\alpha)$ e beta ( $\beta$ ), envolvendo os genes $\alpha$ e $\beta$ globina, respectivamente. ${ }^{4}$ As talassemias alfa se devem à deficiência parcial ou completa da síntese da globina $\alpha$ nas hemácias de indivíduos afetados. ${ }^{5,6}$ As cadeias globínicas $\alpha$ são necessárias para a síntese de hemoglobinas presentes na fase fetal e na fase adulta, exercendo importante papel na manutenção da estabilidade destas moléculas de hemoglobina. Assim, os defeitos que interferem na sua síntese têm repercussão clínica em ambas as fases, diferente das cadeias $\beta$, que estão presentes apenas no componente hemoglobínico adulto maior, a hemoglobina $\mathrm{A}(\mathrm{Hb} \mathrm{A})$.

No feto, a deficiência de globinas $\alpha$ produz um excesso de cadeias gama $(\gamma)$ e, após o sexto mês de vida, o excesso se deve à globina $\beta$, que se tornam livres. Essas cadeias livres formam tetrâmeros denominados de Hb Bart's $\left(\gamma_{4}\right)$ e $\mathrm{Hb} \mathrm{H}\left(\beta_{4}\right)$. A fisiopatologia da talassemia alfa é condicionada, justamente, pela formação desses tetrâmeros, que são instáveis e termolábeis. ${ }^{1,7}$ As talassemias alfa se devem principalmente por defeitos herdados na expressão dos genes que codificam as globinas $\alpha$ atingindo de um a quatro destes genes, ${ }^{5}$ embora, defeitos de síntese também podem ocorrer de forma adquirida. ${ }^{8,9}$ Por essa razão é possível classificar as talassemias alfa em quatro categorias, de acordo com o nível de expressão dos genes $\alpha$ : (1) uma forma assintomática ou portador silencioso, com perda de um único gene $(-\alpha / \alpha \alpha)$; (2) o traço alfa talassêmico, no qual há perda de dois genes alfa de um único cromossomo $(--/ \alpha \alpha)$ ou de um gene $\alpha$ de ambos os cromossomos $(-\alpha /-\alpha)$; (3) a doença da hemoglobina $\mathrm{H}$, na qual apenas um gene alfa é funcional $(--/-\alpha)$ e (4) a hidropsia fetal, caracterizada pela ausência dos quatro genes alfa (--/--). ${ }^{10-13}$ Devido à grande variedade de interações genéticas possíveis, geradas por processos de deleção, não deleção e hemoglobinas variantes, entre outros, pode ocorrer uma sobreposição entre os grupos acima, dificultando a sua caracterização. ${ }^{8} \mathrm{~A}$ anemia presente nas talassemias alfa se deve à diminuição do tempo de sobrevida dos eritrócitos que contêm corpos de inclusão e são, por isto, retirados pela microvasculatura esplenênica. Além disso, pelo defeito na síntese de quantidades normais de hemoglobina, os eritrócitos apresentamse microcíticos e hipocrômicos. ${ }^{7}$ Estes dados são de relevância clínica, uma vez que estas alterações hematológicas são seguidamente interpretadas como indicadores de deficiência de ferro ou características de anemia de doenças

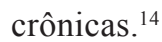

Este estudo teve por objetivo principal estabelecer a freqüência da talassemia alfa e outras hemoglobinopatias em duas populações atendidas no Hospital de Clínicas de Porto Alegre (HCPA): portadores de anemia microcítica não ferropênica a esclarecer (casos) e indivíduos sem anemia atendidos por doenças não hematológicas (controles).

\section{Casuística e Métodos}

O presente trabalho foi realizado após aprovação do Comitê de Ética em Pesquisa do HCPA e pelo Conselho Nacional de Ética em Pesquisa (CONEP) e desenvolvimento, obedecendo integralmente os princípios éticos estabelecidos na Resolução 196/96 do Conselho Nacional de Saúde.

Neste estudo caso-controle, amostras de sangue periférico de 58 casos e 235 controles foram coletadas, após assinatura do Termo de Consentimento Livre Esclarecido (TCLE), no período de março a junho de 2001. Os critérios de inclusão para os casos foram: pacientes acima de 2 anos, atendidos no Ambulatório de Hematologia do HCPA, portadores de anemia microcítica não ferropênica a esclarecer. Para os controles, os critérios de inclusão foram: pacientes acima de 2 anos, não portadores de anemia, atendidos no HCPA por doenças não hematológicas. Foram excluídos do estudo gestantes e pacientes com neoplasias e/ou transplantados.

\section{Procedimentos técnicos}

Os índices hematimétricos (HCT, RBC, VCM, HCM, $\mathrm{CHCM}$ e RDW) e as concentrações de hemoglobina (Hb) foram obtidos de contador eletrônico de células (PentraRoche $\left.{ }^{\circledR}\right)$. A contagem de reticulócitos e a pesquisa citológica de $\mathrm{Hb} \mathrm{H}$ no microscópio foram determinadas após a incubação a $37^{\circ} \mathrm{C}$ durante trinta minutos, de partes iguais de sangue total e azul de cresil brilhante. ${ }^{15}$

A análise qualitativa das frações hemoglobínicas foi realizada em eletroforese em fita de acetato de celulose, $\mathrm{pH}$ 8,6 , com hemolisado preparado a partir de $50 \mu \mathrm{L}$ de sangue total $+100 \mu \mathrm{L}$ de saponina a $1 \% \cdot{ }^{15} \mathrm{~A}$ confirmação e quantificação das $\mathrm{Hb} \mathrm{A}, \mathrm{A}_{2}, \mathrm{~F}, \mathrm{~S}$ e C, além da identificação, quando possível, de $\mathrm{Hb} \mathrm{H}$, foi realizada por sistema automatizado de cromatografia líquida de alta pressão (HPLC) (Bio-Rad Variant ${ }^{\mathrm{TM}}$ - Beta Thal Short Program). ${ }^{16,17}$ Os estoques de ferro foram avaliados através da medida de ferritina sérica, em equipamento de quimioluminescência (Immulite-DPC). Os dados demográficos e clínicos, tais como idade, cor, local de nascimento, história de anemia na família, foram obtidos através de entrevista direta.

\section{Análise estatística}

Os dados foram analisados usando pacotes estatísticos (EPI-INFO versão 6.04b e SPSS versão 6.0). Para comparação das amostras entre as variáveis categóricas (sexo, cor, procedência), recorreu-se ao teste $\chi^{2}$ e ao teste exato de Fisher, quando necessário.

O Teste " $t$ " e a análise de variância (Anova), seguida do Teste de Tukey, foram utilizados para comparação das variáveis quantitativas entre casos e controles e a presença ou não de talassemia alfa. O nível de significância utilizado foi $\mathrm{P}<0,05$. 


\section{Resultados}

Foram estudados 293 casos e controles atendidos no Hospital de Clínicas de Porto Alegre (HCPA), provenientes deste mesmo município e de outras regiões do estado do Rio Grande do Sul, sendo que, do total, $176(60,1 \%)$ eram do sexo feminino e $117(39,9 \%)$ eram do sexo masculino, uniformemente distribuídos entre quatro faixas etárias (Tabela 1).

A presença de anemia e microcitose foi avaliada através dos índices hematimétricos de acordo com o sexo e a faixa etária dos pacientes. ${ }^{18}$ Os resultados do perfil hemoglobínico apresentado pelas análises das amostras estão sumarizados na Tabela 2.

Dentre os 58 casos analisados, a talassemia beta, diagnosticada através do aumento de $\mathrm{Hb} \mathrm{A}_{2}$ e análise dos índices hematimétricos, foi identificada em 19 (32,8\%) amostras. A talassemia alfa, identificada através da presença de $\mathrm{Hb} \mathrm{H}$ na eletroforese em acetato de celulose e pesquisa intraeritrocitária de $\mathrm{Hb} \mathrm{H}$, foi encontrada em 15 (25,9\%) amostras. Destas com talassemia alfa, três $(20 \%)$ apresentaram também a heterozigose para hemoglobina S. Além destas, a heterozigoze para $\mathrm{Hb} \mathrm{S}$ ( $\mathrm{Hb} \mathrm{AS}$ ) e a homozigoze para $\mathrm{Hb} \mathrm{C}$

Tabela 1

Freqüência de casos e controles de acordo com a faixa etária

\begin{tabular}{ccc}
\hline Faixa etária & Casos & Controles \\
\hline $2-6$ anos & $4(6,8 \%)$ & $10(4,3 \%)$ \\
$>6-12$ anos & $7(12,1 \%)$ & $24(10,2 \%)$ \\
$>12-18$ anos & $7(12,1 \%)$ & $28(11,9 \%)$ \\
$>18$ anos & $40(69 \%)$ & $173(76,6 \%)$ \\
\hline Total & $58(100 \%)$ & $235(100 \%)$
\end{tabular}

Tabela 2

Perfil hemoglobínico identificado nas amostras analisadas

\begin{tabular}{cccc}
\hline Perfil Hemoglobínico & Casos $(\mathrm{n})$ & Controles & Total \\
\hline Hb AA & $21(36,2 \%)$ & $202(85,9 \%)$ & 223 \\
Tal $\beta$ & $19(32,8 \%)$ & $2(0,9 \%)$ & 21 \\
Tal $\alpha$ & $12(20,7 \%)$ & $27(11,5 \%)$ & 39 \\
Hb AS & $2(3,4 \%)$ & $3(1,3 \%)$ & 5 \\
Tal $\alpha /$ Hb AS & $3(5,2 \%)$ & $0(0 \%)$ & 3 \\
Hb AC & $0(0 \%)$ & $1(0,4 \%)$ & 1 \\
Hb CC & $1(1,7 \%)$ & $0(0 \%)$ & 1 \\
\hline Total & $58(100 \%)$ & $235(100 \%)$ & 293 \\
\hline
\end{tabular}

$\mathrm{Hb}$ AA: homozigose para hemoglobina A; Tal $\beta$ : talassemia beta; Tal $\alpha$ : talassemia alfa; $\mathrm{Hb}$ AS: traço falciforme; $\mathrm{Hb}$ AC: heterozigose para hemoglobina $\mathrm{C} ; \mathrm{Hb} \mathrm{CC}$ : homozigose para hemoglobina $\mathrm{C}$
( $\mathrm{Hb} \mathrm{CC}$ ) foram identificadas em duas $(3,4 \%)$ e uma $(0,7 \%)$ das amostras analisadas, respectivamente. Assim, dos 58 casos analisados, 37 (63,8\%) apresentaram alguma forma de hemoglobinopatia ou de talassemia.

Entre os 235 controles analisados, $202(85,9 \%)$ apresentaram perfil hemoglobínico normal. Das 33 (14,09\%) amostras alteradas, duas $(0,9 \%)$ apresentaram valores de $\mathrm{Hb} \mathrm{A}_{2}$ aumentados, caracterizando a talassemia beta; 27 (11,5\%) apresentaram a $\mathrm{Hb} \mathrm{H}$ na eletroforese, caracterizando a talassemia alfa; três $(1,3 \%)$ apresentaram a $\mathrm{Hb} \mathrm{S}$ em heterozigoze ( $\mathrm{Hb} \mathrm{AS})$ e uma $(0,4 \%)$ apresentou a $\mathrm{Hb} \mathrm{C}$ em heterozigoze ( $\mathrm{Hb}$ AC). Nenhum caso de aumento de $\mathrm{Hb} F$ foi identificado na amostra.

Estatisticamente, a talassemia alfa identificada entre os casos $(25,9 \%)$ e os controles $(11,5 \%)$ apresentou diferença significativa $(\mathrm{p}=0,0096)$, calculado pelo Teste de $\chi^{2}$, com correção de Yates) com uma razão de chances de 2,69 (I.C. 95\%= $1,23-5,83)$.

A origem racial foi classificada segundo critérios do Instituto Brasileiro de Geografia e Estatística (IBGE), no qual estabelece cinco opções para cores ou raças na nossa população: branca, preta, amarela, parda e índia. ${ }^{19}$ Os dados encontram-se agrupados na Tabela 3.

Através da análise destes dados observamos uma diferença estatística significativa entre casos e controles, com uma maior freqüência de casos de cor preta e parda $(36,8 \%)$ quando comparados com os controles $(19,1 \%)(\mathrm{p}=0,007$, calculado pelo Teste de $\chi^{2}$, com correção de Yates). A mesma variável quando analisada com relação à presença ou não de

Tabela 3

Freqüência da população analisada relativa à origem racial

\begin{tabular}{cccc}
\hline Origem racial & Casos $(n)$ & Controles $(n)$ & Total \\
\hline Branco & $35(61,4 \%)$ & $189(80,4 \%)$ & 224 \\
Negro/Pardo & $21(36,8 \%)$ & $45(19,1 \%)$ & 66 \\
Amarelo & $0(0 \%)$ & $1(0,4 \%)$ & 1 \\
Índio & $1(1,8 \%)$ & $0(0 \%)$ & 1 \\
\hline Total & $57(100 \%)$ & $235(100 \%)$ & 292
\end{tabular}

\begin{tabular}{cccc}
\multicolumn{4}{c}{ Tabela 4} \\
Freqüência da origem racial entre pacientes com \\
e sem talassemia alfa
\end{tabular}

Tal $\alpha$ : talassemia alfa 
talassemia alfa, $26,2 \%$ e $22,0 \%$, respectivamente (Tabela 4), não apresentou diferença estatística $(p=0,69)$.

Os índices hematimétricos apresentados pelos pacientes com e sem talassemia alfa foram avaliados através da análise de variância (Anova), seguida do Teste de Tukey, considerando a faixa etária ao qual pertenciam (Tabela 5). Nesta análise, os valores de HCT, Hb, VCM, HCM e CHCM foram menores no grupo de pacientes com talassemia alfa quando comparados com o grupo de pacientes sem talassemia alfa, apresentando diferença estatística significativa $(\mathrm{P}<0,05)$ os seguintes índices: HCT, Hb, VCM e HCM em pacientes $>18$ anos.

\section{Discussão}

A população brasileira se caracteriza por apresentar uma grande heterogeneidade genética e um nível de miscigenação significativo e progressivo. ${ }^{20} \mathrm{~A}$ distribuição das hemoglobinopatias e talassemias se relaciona com os diversos grupos raciais que participaram na formação da população brasileira. O IBGE estabelece cinco opções para cor ou raça na nossa população: branca, preta, amarela, parda e índia. Os dados do censo realizado em 2000 apresentam a classificação da população brasileira de acordo com esta variável. Indivíduos de cor preta ou parda representam $45 \%$ da população brasileira, enquanto esta mesma categoria no estado do Rio Grande do Sul representa $13 \% .{ }^{19}$ No grupo estudado, identificamos a presença de $36,8 \%$ de casos e $19,1 \%$ de controles classificados nestas categorias. Esta proporção maior de pacientes destas categorias com relação ao estado do Rio Grande do Sul pode ser devido à possível seleção da população que procura assistência médica no HCPA, onde predomina o atendimento pelo Sistema Único de Saúde, de indivíduos economicamente menos favorecidos, onde o número de afro-descendentes é maior. ${ }^{21}$ Quando verificamos a freqüência da cor preta e parda com e sem talassemia alfa na amostra analisada, observamos $26,2 \%$ e $22,0 \%$ (Tabela 4), respectivamente nestas categorias, sendo esta diferença estatística não significativa. Isto pode sugerir um processo de mistura racial na população de Porto Alegre, na qual a presença de genes africanos e europeus é aproximadamente semelhante. ${ }^{22}$

Os resultados apresentados nesse trabalho demonstram que as talassemias e as hemoglobinopatias são muito freqüentes em populações de indivíduos com anemia microcítica não ferropênica $(63,8 \%)$. Com relação à talassemia alfa, a sua freqüência verdadeira não é definida, uma vez que a tecnologia utilizada não é capaz de detectar todos os portadores. ${ }^{10}$ Diversos estudos realizados têm demonstrado a grande variação na proporção de indivíduos com talassemia alfa no Brasil e no mundo. ${ }^{10,20,21,23-27}$ Esta variação encontrada nas populações estudadas pode ser devido a: (1) real diferença nas populações e (2) uso de técnicas diferentes. Na região de São José do Rio Preto, Castilho et al identificaram, através de técnicas citológicas, uma prevalência maior (16\%) de portadores de talassemia alfa em indivíduos com anemia a esclarecer quando comparada com indivíduos sem anemia (4\%). ${ }^{28} \mathrm{Em}$ Campinas, dos 47 doadores de sangue negros estudados através de biologia molecular, 29,8\% 
demonstraram ser portadores desta mesma condição. ${ }^{29}$

A alta freqüência de talassemias alfa e beta identificada entre os casos pode ser devido ao Ambulatório de Hematologia do HCPA constituir-se em centro de referência no estado para diagnóstico e tratamento de distúrbios hematológicos. Além disso, a deficiência de ferro e as anemias de doenças crônicas, causas de microcitose, foram excluídas através de exames laboratoriais e consultas médicas. Já a freqüência de talassemia alfa entre os controles pode sugerir que estes sejam apenas portadores silenciosos, uma vez que não apresentam alterações em nível hematológico. Nossos resultados são consistentes com a observação de que muitos portadores de deleções que atingem um único gene possuem estes índices nos níveis de normalidade. $^{30}$

Quando analisamos os resultados obtidos por Daudt, em 2002 (0,12\% de Hb Bart's em recém-nascidos no HCPA), verificamos que este valor é bastante inferior ao encontrado neste trabalho. Justifica-se este baixo índice pela técnica utilizada, uma vez que as amostras foram coletadas em papel filtro e foram processadas, em média, 22 dias após a coleta, o que possivelmente prejudica a identificação de hemoglobinas com mobilidade mais rápida do que a $\mathrm{Hb} \mathrm{A.}{ }^{21,31}$

Com as técnicas utilizadas na realização deste trabalho, grande número de pacientes com anemia microcítica não ferropênica a esclarecer pode ser diagnosticado como portador de algum tipo de anemia hereditária, destacando as talassemias alfa e beta. A principal vantagem da identificação correta destes indivíduos é evitar a administração desnecessária e deletéria de ferro, além de exames laboratoriais e consultas médicas. Destaca-se, porém, a presença de pacientes sem anemia, com índices hematimétricos normais, classificados como portadores silenciosos de talassemia alfa.

Embora as técnicas aplicadas neste estudo tenham sido capazes de diagnosticar, aproximadamente, dois terços de pacientes com anemia não ferropênica, o restante dos pacientes apenas será diagnosticado através do uso de técnicas de biologia molecular, ainda não disponíveis para uso na rotina da maioria dos laboratórios clínicos. Para a talassemia alfa, a identificação de $\mathrm{Hb} \mathrm{H}$ realizada através das técnicas empregadas neste trabalho, se mostrou útil, relativamente simples, reprodutível e de baixo custo.

\section{Abstract}

To establish the frequency of hemoglobinopathies and thalassemias in patients with non-ferropenic anemia, 58 patients with confirmed non-ferropenic anemia and 235 non-anemic individuals (control group) were studied. All samples were obtained from the Hospital de Clínicas de Porto Alegre (HCPA), Rio Grande do Sul, Brazil. The techniques used were Alkaline pH cellulose acetate electrophoresis and cytological screening of $\mathrm{Hb}, \mathrm{Hl}, \mathrm{HPLC}$, hemogram and ferritin. The data analysis showed that $63 \%$ of the patients with nonferropenic anemia carried some type of inherited anemia: $25.9 \%$ of heterozygous $\alpha$-thalassemia, 32.8\% of heterozygous $\beta$-thalassemia, $3.4 \%$ of heterozygosity for hemoglobin $\mathrm{S}(\mathrm{Hb} \mathrm{AS})$ and $1.7 \%$ of homozygosity for hemoglobin $\mathrm{C}$ ( $\mathrm{Hb} \mathrm{CC}$ ). Inherited anemias were detected in $14.1 \%$ of the control group: $11.5 \%$ of $\alpha$-thalassemia, $0.9 \%$ of $\beta$-thalassemia, $1.3 \%$ of heterozygosity for hemoglobin $S$ $(\mathrm{Hb} \mathrm{AS}$ ) and $0.4 \%$ of heterozygosity for hemoglobin $\mathrm{C}$ ( $\mathrm{Hb} \mathrm{AC}$ ). The results obtained showed that the prevalence of variant thalassemias and hemoglobins in the control group is coincident with that described in the literature. However, physicians and health services should be informed about the overwhelming prevalence of these inherited homeopathies in individuals with non-ferropenic anemia, due to its importance in the definitive diagnosis of anemia and for the correct therapeutic proceedings.Rev. bras. hematol. hemoter. 2005;27(1):37-42.

Key words: Hemoglobinopathies; thalassemia; non-ferropenic anemia; epidemiology.

\section{Agradecimentos}

Agradecemos à bioquímica Ana Stela Goldbeck e demais funcionários do Laboratório de Triagem Neonatal da Faculdade de Farmácia - UFRGS, pelo auxílio nas determinações realizadas no equipamento de HPLC, e à Dra. Vânia Hirakata, pelo auxílio na análise estatística. Agradecemos também à Bio-Rad Latin America (EUA) e Bio-Oxford Importação (São Paulo/SP) pela doação de reagentes utilizados no equipamento de HPLC.

\section{Referências Bibliográficas}

1. WHO Working Group. Hereditary anaemias: genetic basis, clinical features, diagnosis, and treatment. Bull WHO 1982; 60: 643-60.

2. Angastiniotis M, Modell B, Englezoz P et al. Prevention and control of haemoglobinopathies. Bull WHO 1995; 73: 375-86.

3. Globin Gene Server Home Page. http://globin.cse.psu.edu (acessado em $13 / \mathrm{Jan} / 2005)$.

4. Weatherall DL, Clegg JB. Inherited disorders of haemoglobin. The hemoglobinopathies. In: Weatherall DJ, Clegg JB. The thalassaemia syndromes. London: Blackwell Scientific Publications; 1981. p. $85-132$.

5. Weatherall DL, Clegg JB. The $\alpha$ thalassaemias. In: Weatherall DJ, Clegg JB. The thalassaemia syndromes. London: Blackwell Scientific Publications; 1981. p. 508-612.

6. Waye JS, Chui DHK. The $\alpha$-globin gene cluster: genetics and disorders. Clin Invest Med 2001;24:103-9.

7. Weatherall DJ. The thalassemias. In: Beutler E, Lichtman MA, Coller B, Kipps TJ, Seligsohn U, editores. Williams Hematology, 6 ed. New York: McGraw-Hill; 2001. p. 547-78.

8. Liebhaber S. $\alpha$ Thalassemia. Hemoglobin 1989;13:685-731.

9. Weatherall DJ, Old J, Longley J et al. Acquired haemoglobin H disease in leukaemia: pathophysiology and molecular basis. $\mathrm{Br} \mathrm{J}$ Haematol 1978; 38:305-22.

10. Pedrollo E, Hutz MH, Salzano FM. Alpha thalassemia frequency in newborn children from Porto Alegre, Brazil. Rev Brasil Gen 1990; 13:573-81.

11. Kazazian HH. The thalassemia syndromes: molecular basis and prenatal diagnosis in 1990. Semin Hematol 1990;27:209-28.

12. Chui DHK, Waye JS. Hydrops fetalis caused by $\alpha$-thalassemia: an emerging health care problem. Blood 1998;91:2213-22.

13. Lafferty JD, Crowther MA, Waye JS et al. A reliable screening test to identify adult carriers of the (-- $\left.{ }^{\mathrm{SEA}}\right)$ alpha ${ }^{0}$ - thalassemia deletion. Am J Clin Pathol 2000;114:927-31. 
14. Brittenham GM. Disorders of iron metabolism: iron deficiency and overload. In: Hoffman R, Benz Jr EJ, Shattil SJ, Furie B, Cohen HJ, Silberstein LE et al, editores. Hematology - Basic Principles and Practice. New York: Churchill Livingstone; 2000. p. 397-426.

15. Naoum PC, Bonini-Domingos CR. Técnicas laboratoriais para identificação das hemoglobinas normais e anormais. In: Naoum PC. Hemoglobinopatias e Talassemias. São Paulo: Savier Editora. Livros Médicos; 1997. p. 144-70.

16. Fucharoen $\mathrm{S}$ et al. Prenatal and postnatal diagnoses of thalassemias and hemoglobinopathies by HPLC. Clin Chem 1998;44:740-48.

17. Clarke GM, Higgins TN. Laboratory investigation of hemoglobinopathies and thalassemias: review and update. Clin Chem 2000;46:1284-90

18. Lanzkowsky P. Manual of Pediatric Hematology and Oncology. $2^{\mathrm{a}}$ ed. New York: Churchill Livingstone; 1995.

19. Fundação Instituto Brasileiro de Geografia e Estatística. Censo Demográfico 2000. http://www.ibge.gov.br (acessado em 21/Ago/ 2004)

20. Orlando GM, Naoum PC, Siqueira FAM et al. Diagnóstico laboratorial de hemoglobinopatias em populações diferenciadas. Rev Bras Hematol Hemot 2000;22:111-121.

21. Daudt LE, Zechmaister D, Portal L et al. Triagem neonatal para hemoglobinopatias: um estudo piloto em Porto Alegre, Rio Grande do Sul, Brasil. Cad Saúde Pública 2002;18:833-41.

22. Bortolini MC, Silva WAJR, Guerra DS et al. African-derived South American populations: A history of symmetrical and asymmetrical matings according to sex revealed by bi- and uni-parental genetic makers. Am J Hum Biol 1999;11:551-63.

23. Velati C, Sampietro M, Biassoni M et al. Alpha Thalassaemia in an Italian population. Br J Haematol 1986;63:497-501.

24. Naoum PC, Isiqui WD, Pagotto RC et al. Prevalência de hemoglobinopatias em amostras de proveniência diferenciada. Bol Soc Bras Hematol Hemoter 1989;153:69-72.
25. Peres MJ, Carreiro MH, Machado MC et al. Neonatal screening of hemoglobinopathies in a population residing in Portugal. Acta Med Port 1996;9:135-39.

26. Viana-Baracioli LMS, Bonini-Domingos CR, Pagliusi RA et al. Prevenção de hemoglobinopatias, a partir do estudo em gestantes. Rev Bras Hematol Hemoter 2001;23:31-39.

27. Ducatti RP, Teixeira AEA, Galão HE et al. Investigação de hemoglobinopatias em sangue de cordão umbilical de recém-nascidos do Hospital de Base de São José do Rio Preto. Rev Bras Hematol Hemoter 2001;23:23-9.

28. Castilho EM, Naoum PC, Graciano RA et al. Prevalência de talassemia alfa em pacientes com anemia e em pessoas sem anemia. Rev Patol Clin 1987;23:131-34.

29. Sonati MF, Farah SB, Ramalho AS et al. High prevalence of $\alpha$ Thalassemia in a black population of Brazil. Hemoglobin 1991; 15:309-11.

30. Chan LC, Ma SK, Chan AYY et al. Should we screen for globin gene mutations in blood samples with mean corpuscular volume (MCV) greater than $80 \mathrm{fl}$ in areas with a high prevalence of thalassaemia? J Clin Pathol 2001;54:317-20.

31. Kutlar A, Ozcan O, Brisco JT et al. The detection of hemoglobin variants by isoelectrofocusing using EDTA-collected and filter paper-dried cord blood specimens. Am J Clin Pathol 1990; 94: 199-202.

Avaliação: Editor e dois revisores externos

Conflito de interesse: não declarado

Recebido: 28/02/05

Aceito após modificações: 28/03/05 